\title{
Electromagnetic MetaMaterial Insulator To Eliminate Substrate Surface Waves
}

\author{
Kevin Buell ${ }^{*}$, Hossein Mosallaei, and Kamal Sarabandi \\ Radiation Laboratory \\ Department of Electrical Engineering and Computer Science \\ The University of Michigan, Ann Arbor, MI 48109-2122 \\ kbuell@umich.edu, hosseinm@umich.edu, sarabandi@umich.edu
}

\begin{abstract}
Metamaterial EM insulators are designed to eliminate mutual coupling between closely packed array elements. This technique allows for array element design in isolation, without consideration of mutual coupling effects. Eliminating mutual coupling also allows for denser packing and higher directivity in antenna arrays. By placing Isolation Walls along the radiating edges of a patch on a $\lambda_{0} / 3 \times \lambda_{0} / 3$ groundplane a $19.2 \mathrm{~dB}$ front-toback ratio is achieved. Metamaterial Isolation walls $0.05 \lambda_{0}$ thick exhibit $-10 \mathrm{~dB}$ isolation bandwidths of $2-6 \%$ with very low losses for various geometries and greater than $20 \mathrm{~dB}$ peak isolation is achieved for array element spacing of $0.2 \lambda_{0}$.
\end{abstract}

\section{Introduction}

Metamaterial EM insulators are formed by embedded circuit metamaterials operating in a band-gap region and can dramatically reduce mutual coupling between densely packed adjacent elements. A single $0.05 \lambda_{0}$ thick layer of embedded circuit MetaMaterial insulators placed between radiating elements achieves better than $-20 \mathrm{~dB}$ S21 coupling improvement, effectively eliminating the mutual coupling between all but the most densely spaces array elements.

Densely packed antenna arrays enticingly offer high gain with small size. While mathematically intriguing and long a popular topic of theoretical papers [1-2] close-packed, high gain arrays have generally been impractical for two reasons. In order to achieve high directivity, adjacent elements are of alternating sign (Table 1) and the net effective current which produces radiation at broadside is resultingly quite low, yielding very little radiated power. Consequently, to achieve reasonable radiated power levels the excitations currents are generally quite high and this is the source of the two reasons that superdirective antenna arrays are generally impractical, even for narrow bandwidths. First, the high current amplitudes result in high ohmic losses. The low radiated power level despite high current amplitudes indicates a low radiation resistance- sometimes even lower than the ohmic resistances of the radiating elements for aggressively small arrays. Second, the high precision required in controlling element current levels becomes impossible for closely packed elements due to mutual coupling.

There are various techniques for addressing the ohmic losses discussed, including superconducting antennas which result in near $100 \%$ antenna efficiency [3] and judicious choice of excitation coefficient array functions which yield $30 \%$ efficiency. [4]

\section{Theory}

Embedded Circuit MetaMaterials provide highly efficient bandstop rejection. Typical geometry for a single resonant element is shown in Figure 1.[5] These single elements resonators are stacked to form solenoidal isolation walls which surround the radiating array elements (fig 2). Fabrication of the spiral geometry was achieved by standard commercial etching of $1 / 2 \mathrm{Oz}$ copper on 0.125 " thick Rogers RT/duroid $5880\left(\varepsilon_{\mathrm{T}}=2.2\right.$, Tan $\delta=9 \mathrm{E}-4)$.

The spiral geometry of the embedded circuit metamaterial provides a passive LC-resonant behavior which blocks the transmission of EM energy in the frequency band around the resonance frequency by exhibiting an extremely high wave impedance. Figure 2 shows a typical transmission and reflection at normal incidence through an infinite sheet one layer thick, as simulated by a commercial FEM tool (HFSS). Better than $20 \mathrm{~dB}$ isolation is achieved by a single $0.05 \lambda_{0}$ thick isolation layer. 
This metamaterial isolation wall is low-loss and highly compact, implying many promising applications. For the purpose of this project we will consider their implementation to eliminate mutual coupling between adjacent radiating elements of a densely packed antenna array.

When one attempts to densely pack planar antennas such as rectangular microstrip patch antennas, the mutual coupling between adjacent elements is dominated by trapped waves traveling along the substrate surface. This coupled energy makes input matching and element current excitation control extremely difficult. If mutual coupling can be eliminated, significant improvement in array design may be achievable. By separating array elements with metamaterial isolation walls, the individual array elements may be designed in isolation without considering mutual coupling- a significant improvement in design and potentially a boon for array designers. In the region around the isolator resonance the isolation wall behaves similar to a perfect magnetic conductor (PMC), providing a reasonably matchable impedance to the nearby patch without the extreme loss effect that a matched absorber would produce when in the near field of a high $Q$ radiating antenna. In fact, near-perfect radiating efficiency is achieved for a single radiating patch surrounded by isolation walls- indicated extremely low isolator wall losses.

\section{Results}

A single patch situated between two isolation walls is the basic array element. Design and simulation on a typical desktop PC $(1.8 \mathrm{GHz})$ was quite reasonable and achieved in four hours with commercially available FEM code (HFSS). For an aggressively miniaturized $0.11 \lambda_{0}$ patch on a thick $\left(0.051 \lambda_{0}\right)$, low-loss dielectric substrate $\left(\varepsilon_{\mathrm{r}}=15\right.$, Tan $\delta_{\mathrm{e}}=2 \mathrm{E}-4,1.4$ mil thick copper $)$, better than $95 \%$ radiation efficiency was achieved and the E-plane radiation pattern is given in Figure 11 .

To test the isolation capabilities of the embedded circuit isolation wall between patch elements an in-house FDTD code was employed to model the two-patch geometry of Fig.6. This FDTD code approximates all conductors as PEC's, so is not suitable for efficiency calculations of highly resonant circuits. Without the isolation wall present, the close-packing of array elements causes extremely strong mutual coupling due to substrate trapped surface waves (Fig. 7). When the isolation wall is inserted to this ideal simulation, an astonishing $40 \mathrm{~dB}$ improvement in mutual coupling is observed with over $6 \%-10 \mathrm{~dB}$ bandwidth from a single isolation layer (Fig. 8).

A single Isolation wall in Figure 4 incorporates thirteen embedded circuit isolators, and for arrays the simulation complexity quickly becomes unmanageable for HFSS simulation. To simplify simulation complexity the polarization dependant equivalent permittivity and permeability of the isolation walls were determined and are shown in Figure 3. Blocks with these effective material properties may be used to represent isolation walls, making simulation of larger arrays possible.

A five-element array with a total length of $1.18 \lambda_{0}$ was simulated in HFSS using this equivalent material to represent the isolation walls (Fig 9). The return loss and adjacent element coupling of the five elements are shown in Fig. 10. Since the radiation conditions of the various patches are not exactly identical slight deviation in their scattering parameters are observed but this is at least partly due to imperfect simulation convergence. Under uniform excitation conditions the array exhibits gain of $+6 \mathrm{dBi}, 142$ degree first-null beamwidth and $81 \%$ radiation efficiency.

By hand-tuning of excitation coefficients, a superdirective E-plane pattern was achieved that demonstrates the enhanced directivity possible with array element coupling isolation. This hand-tuned array exhibits a gain of $-2.5 \mathrm{dBi}, 90$ degree beamwidth and $64 \%$ radiation efficiency. A more rigorous optimization of excitation coefficient by genetic algorithm will be investigated for conference.

\section{Challenges and Continuing Work}

Although the radiating elements are effectively isolated from each other, the isolation wall itself interacts with the trapped substrate surface waves. A portion of this scattered energy is directed broadside and this affects the far-field pattern. This scattered energy must be accounted for in the choice of excitation coeffiecients. For this reason, a simple and direct implementation of the Dolf-Chebychev polynomials or similar array factor optimization technique is not satisfactory. Instead, once the near-field response to excitation at the input ports has been identified by HFSS simulation, the optimal excitation coefficients may be selected by genetic algorithm without further time consuming simulation.

The isolation walls and patch radiating elements have been physically fabricated and verified. Once optimal excitation coefficients have been identified by means of genetic algorithm, the feed network will be fabricated and the final array can be measured for presentation at conference. 


\section{Figures}

\section{$0.05 \lambda_{0}$}

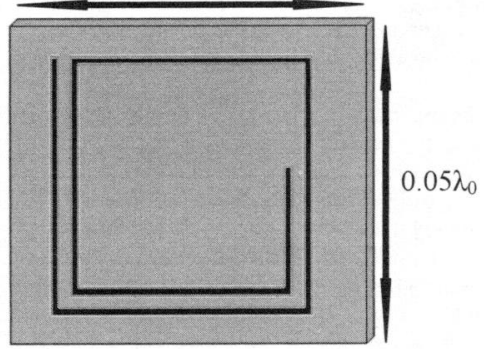

Figure 1: Embedded Circuit Resonating Isolator.

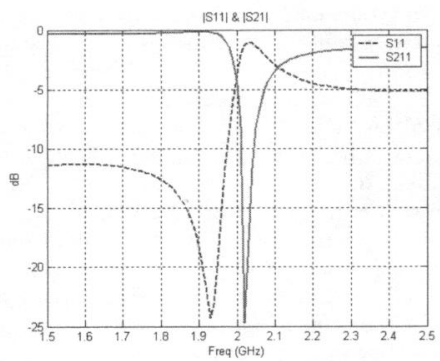

Figure 2: Transmission and Reflection Coefficient Through Embedded Circuit Resonating Isolator Wall.

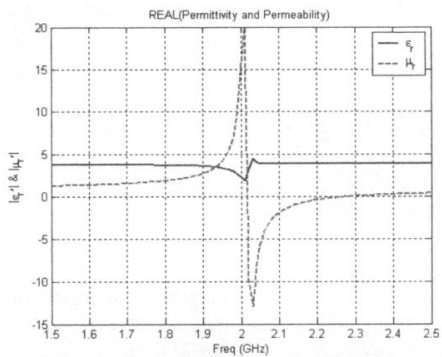

Figure 3A: Real Effective Permittivity and Permeability of Embedded Circuit Metamaterial Resonating Isolator Wall .

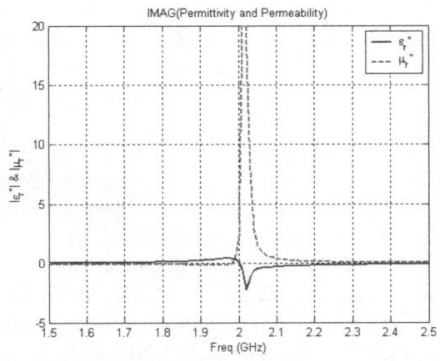

Figure 3B: Imaginary Effective Permittivity and Permeability of Embedded Circuit Metamaterial Resonating Isolator Wall .

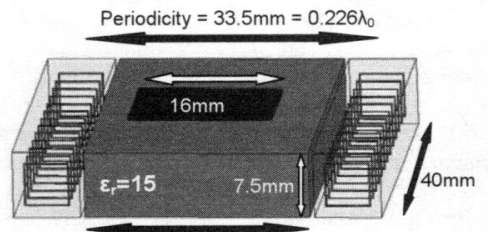

$25 \mathrm{~mm}$

Figure 4: Antenna A: Patch Antenna with Embedded Circuit Isolator Walls.

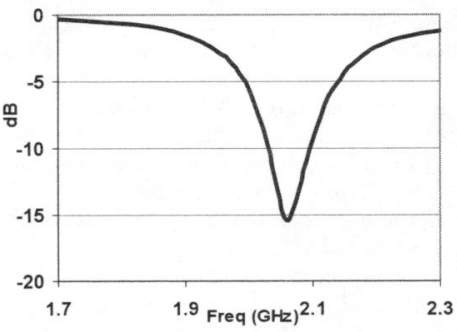

Figure 5: Input Matching of Antenna A

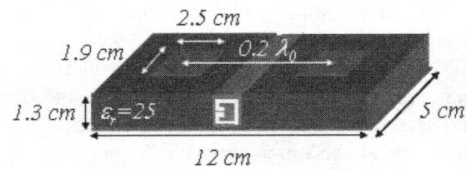

Figure 6: Antenna B, $0.2 \lambda_{0}$ Peridicity 


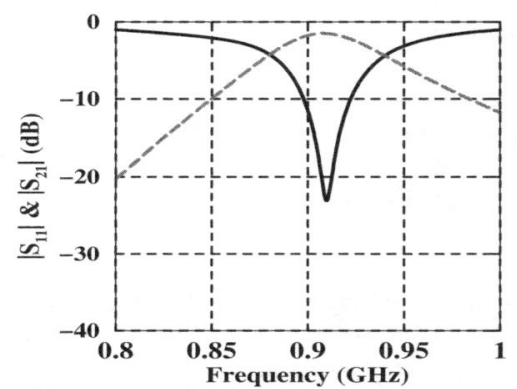

Figure 7: S11 and S21 of Antenna B, two adjacent patches with $0.2 \times \lambda_{0}$ periodicity without Metamaterial isolation wall

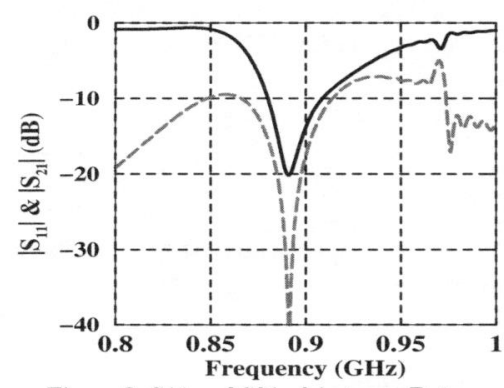

Figure 8: S11 and S21 of Antenna B, two adjacent patches with $0.2 \times \lambda_{0}$ periodicity with Metamaterial isolation wall

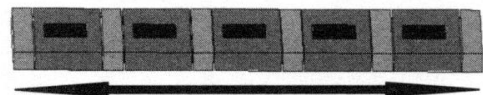

$1.18 \lambda_{0}$

Figure 9: Antenna Array C, 5 Element Patch Array of Antenna A with $0.226 \times \lambda_{0}$ Peridicity

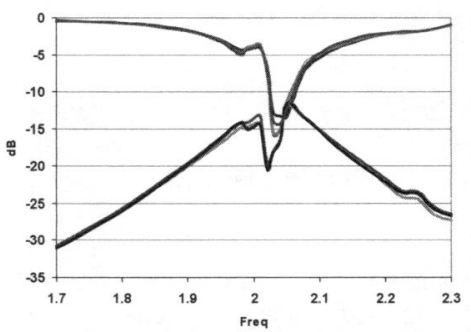

Figure 10: Antenna Array C Input matching and Coupling with ECM Isolators

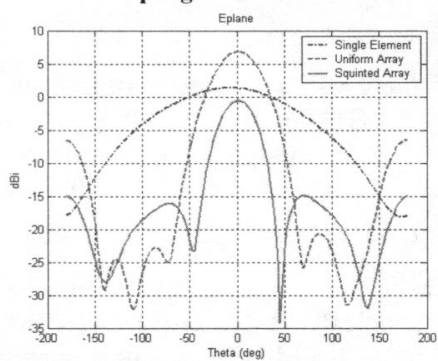

Figure 11: Antenna $A$ and $C$ E-plane Radiation Patterns for various uniform and squinted excitation coefficients

\begin{tabular}{|l|l|l|}
\hline & Uniform & $\begin{array}{l}\text { Squint- } \\
\text { Beam }\end{array}$ \\
\hline I1 & 1.000 & 0.508 \\
\hline I2 & 1.000 & -0.292 \\
\hline I3 & 1.000 & 1.0000 \\
\hline I4 & 1.000 & -0.292 \\
\hline I5 & 1.000 & 0.508 \\
\hline
\end{tabular}

Table 1: Excitation Coefficients for Antenna Array C

\section{References:}

[1] C. L. Dolph, "A Current Distribution for Broadside Array Which Optimizes the Relationship Between Beamwidth and Sidelobe Level," Proc. IRE., vol. 34, no. 6, 1946, p. 335.

[2] A. Bloch et al., "Superdirectivity," Proc. IRE., vol. 48, 1960, p. 1164.

[3] G. B. Walker et al, "Superconducting Superdirectional Antenna Arrays," IEEE Trans. on Antennas and Propagation, vol. 25, no: 6, 1977, p:885

[4] M. M. Dawoud et al "Design and Simulation Of Superdirective Adaptive Antenna Arrays," 1990 IEEE Antennas and Propagation Symposium, AP-S. Digest p.1700-1703 vol.4

[5] K. Buell et al "Embedded-circuit magnetic metamaterial substrate performance for patch antennas," Antennas and Propagation Society Symposium, 2004. IEEE ,Volume: 2, 20-25 June 2004, Pages:1415 $1418 \mathrm{Vol} .2$

[6] O, Ishii et al, "Miniaturization of array antennas with superdirective excitation," 1993 IEEE

Antennas and Propagation Symposium, AP-S. Digest, 28 June-2 July 1993 Pages:1850 - 1853 vol.3 